\title{
Ethylene-styrene copolymerization with constrained geometry catalysts: A density functional study
}

Cite as: J. Chem. Phys. 122, 074901 (2005); https://doi.org/10.1063/1.1845393

Submitted: 04 June 2004 . Accepted: 15 November 2004 . Published Online: 07 February 2005

Javier Ramos, Antonio Muñoz-Escalona, Sonia Martínez, Javier Martínez-Salazar, and Victor Cruz

\section{ARTICLES YOU MAY BE INTERESTED IN}

Density functional study of the insertion mechanism for ethylene-styrene copolymerization with constrained geometry catalysts

The Journal of Chemical Physics 119, 1824 (2003); https://doi.org/10.1063/1.1581254

\section{Lock-in Amplifiers up to $600 \mathrm{MHz}$}
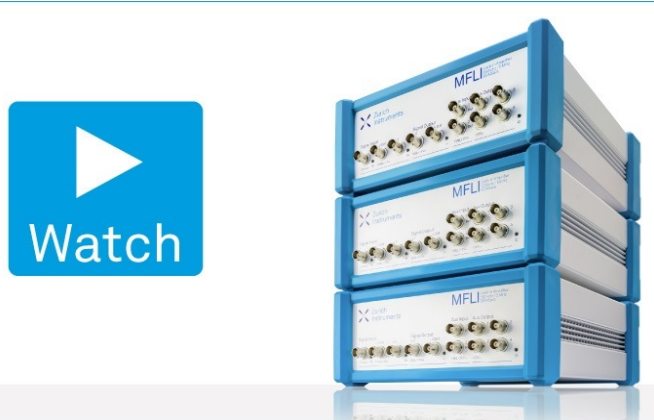

J. Chem. Phys. 122, 074901 (2005); https://doi.org/10.1063/1.1845393

122, 074901

(c) 2005 American Institute of Physics. 


\title{
Ethylene-styrene copolymerization with constrained geometry catalysts: A density functional study
}

\author{
Javier Ramos, Antonio Muñoz-Escalona, Sonia Martínez, and Javier Martínez-Salazara) \\ Departamento de Física Macromolecular, Instituto de Estructura de la Materia, CSIC, Serrano \\ 113bis, 28006 Madrid, Spain \\ Victor Cruz \\ Centro Técnico de Informática, CSIC, Pinar 19, 28006 Madrid, Spain
}

(Received 4 June 2004; accepted 15 November 2004; published online 7 February 2005)

\begin{abstract}
A density functional theory (DFT) study of the ethylene-styrene copolymerization process with titanium-based constrained geometry catalyst (CGC) is presented. To establish the difference between simplified CGC or real CGC models, i.e., considering all ligands of the catalyst, we have performed calculations for ethylene and styrene insertions in both models. Thus, we have used two different DFT functional, BP86 and B3L YP along with two basis set, LANL 2DZ (without polarization functions) and DZVP (including polarization functions). We have noted certain differences between theoretical results published by other authors and our theoretical and experimental data. (C) 2005 American Institute of Physics. [DOI: 10.1063/1.1845393]
\end{abstract}

\section{INTRODUCTION}

Homogeneous single-site catalyst systems based on zirconium and titanium organometallic compounds activated by methylaluminoxane (MAO) or other cocatalysts (borates) have been used for ethylene-styrene copolymerization. Depending on the catalyst structure and the polymerization reaction conditions (i.e., the ethylene/styrene concentration ratio in the reactor feed) a broad variety of materials can be produced. Recently, we have performed experimental and computational studies in order to clarify the mechanisms involved in the ethylene-styrene copolymerization with metallocene, half-sandwich, and constrained geometry catalysts. $^{1-4}$

A density functional (DFT) study of ethylene-styrene copolymerization mechanisms based on the simplified constrained geometry catalyst (CGC) model $\left.\left[\mathrm{H}_{2} \mathrm{Si}\left(\mathrm{C}_{5} \mathrm{H}_{4}\right) \mathrm{NCH}_{3}\right) \mathrm{Ti}-\mathrm{R}\right]^{+}$(where $\mathrm{R}$ is an alkyl chain), hereafter denoted as simplified CGC has been reported by Yang et al..$^{5}$ In this work, the insertion monomer process was monitored by a fixed stepwise decrease in the reaction coordinate, optimizing the energy with respect to all other degrees of freedom at each step (this method is usually known as "coordinate driving"). The reaction coordinate selected was the distance between the carbon atom attached to the titanium center $\mathrm{C}_{\alpha}$ and the closest carbon atom in the monomer $\mathrm{C}_{2}$.

The main aim of the present paper is twofold. On the one hand, we will compare the theoretical results with the experimental findings for the ethylene-styrene copolymerization. And on the other, we will evaluate the effect of some computational strategies on the calculated monomer coordination and energy barriers. The computational strategies here examined are the following: (a) Differences between considering a

\footnotetext{
a) Author to whom correspondence should be addressed. Fax: +34915855413. Electronic mail: jmsalazar@iem.cfmac.csic.es
}

"simplified" model or a "real" model for the CGC catalyst, (b) the effect of the use of polarization functions into the calculations, and (c) the choice of the method for locating transition state structures on the potential energy surface for the insertion process.

\section{COMPUTATIONAL METHODS}

Computational methods are very similar to our previous works. ${ }^{3,4,6,7}$ Geometry, energy, and vibrational frequency calculations were made using BP86 and B3LYP DFT models. All calculations were performed using the GAUSSIAN 98 package. ${ }^{8}$ Two basis set were used in this work: (a) The LANL2DZ basis set which does not consider polarization functions and (b) the DZVP basis set which includes polarization functions for all but hydrogen atoms. For more details see Refs. 9 and 10, respectively.

Transition state geometries were obtained by the STQN (synchronous transit-guided quasi-Newton) method to locate an estimate saddle-point in the path from reactant to product. ${ }^{8,11}$ Subsequently, this saddle point was completely optimized following the negative eigenvector. Frequency calculations were performed to check the nature of the identified stationary points. Transition states were characterized by exactly one imaginary frequency, visualizing the corresponding eigenvector.

For the present calculations, the role given to the cocatalyst MAO is to create the active site by alkylating the catalyst precursor and removing one of the methyl group. The cationic titanium species, with a vacant site in the coordination sphere, was thus taken as the starting point for the polymerization reactions with the monomers.

\section{RESULTS AND DISCUSSION}

The mechanism of ethylene-styrene copolymerization was followed according to Fig. 1. For the sake of clarity and 




FIG. 1. Active species and mechanism steps for the ethylene and styrene copolymerization process using the constrained geometry catalysts. E and St stand for ethylene and styrene monomers, respectively.

briefness, all styrene insertions are considered to be secondary. This assumption is based on the general acceptance that secondary styrene insertion is the preferred mechanism of styrene polymerization for an ansa-metallocene-based catalyst. However, this assumption might not be valid for a CGC-based catalyst. Indeed, our theoretical calculations suggest that primary and secondary styrene insertions are practically competitive in CGC-based catalysts. ${ }^{1}$

To establish the difference between simplified CGC or real CGC models, i.e., considering all ligands of the catalyst (see Fig. 2), first we have performed calculations for ethylene (E) and styrene (St) insertions into both catalysts. We considered two theoretical models, B3LYP and BP86, along with the LANL2DZ basis set. Table I summarizes the results for the ethylene-styrene copolymerization process using LANL2DZ basis set. We have also performed calculations using a polarized basis set (DZVP). ${ }^{10}$ The inclusion of polarization functions yields differences below $2 \mathrm{kcal} / \mathrm{mol}$ with
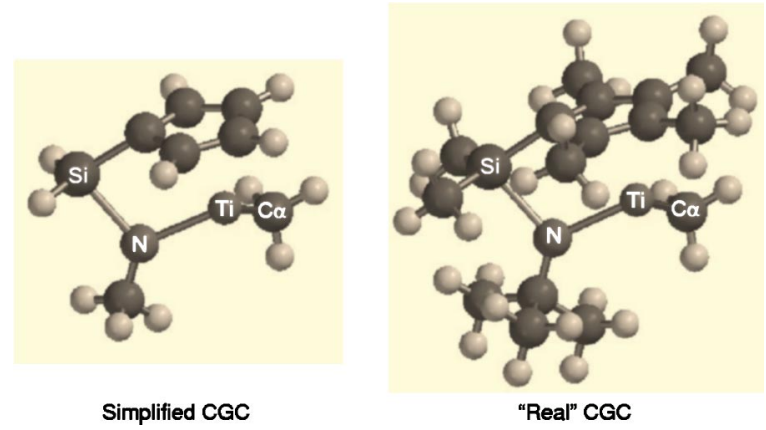

FIG. 2. Simplified and real models for the constrained geometry catalysts based on titanium atom (Ti-CGC).

respect to the method using LANL2DZ in both complexation energies and energy barriers as can be seen in Table II. These results are in agreement with other authors who have reported that the use of a basis set extended with polarization functions, do not greatly affect the ethylene insertion energy barriers in similar organometallic catalyts. ${ }^{12,13}$

In order to establish the activity of the catalyst, it is commonly accepted to relate it with the insertion energy barriers. The complexation energies play a secondary role in the activity of the catalyst. The main difference found when comparing energy barriers corresponding to $\mathrm{E}$ and St insertions for the simplified CGC and real CGC models, is a relatively lower complexation energies for the real CGC system. However, electronic energy barriers using B3LYP/ LANL2DZ, BP86/LANL2DZ, and BP86/DZVP, methods are

TABLE I. Results for the ethylene and secondary styrene monomer insertion into the Ti-Me, Ti-E and Ti-St species for the CGC-simplified and CGC-real catalysts at BP86 level obtained by Yang et al. and by us. Enclosed between parentheses are the results corresponding to B3LYP/LANL2DZ level. Energies, negative frequencies, and distances corresponding to transition structures are given in $\mathrm{kcal} / \mathrm{mol} \mathrm{cm}^{-1}$, and angstroms, respectively. ( n.a., not appreciable; n.d., not determined).

\begin{tabular}{|c|c|c|c|c|c|c|c|c|}
\hline & \multirow[b]{2}{*}{ Species $^{\mathrm{b}}$} & \multirow[b]{2}{*}{ Monomer $^{\mathrm{b}}$} & \multicolumn{2}{|c|}{$\Delta E_{\text {comp }}{ }^{\mathrm{c}}$} & \multicolumn{2}{|c|}{$\Delta E^{\mathrm{a}}$} & \multirow{2}{*}{$\begin{array}{l}\text { Imaginary } \\
\text { frequency }^{\mathrm{a}}\end{array}$} & \multirow[b]{2}{*}{$\mathrm{C}_{2}-\mathrm{C}_{\alpha}{ }^{\mathrm{a} f}}$, \\
\hline & & & Ref. $5^{\mathrm{e}}$ & This work ${ }^{\mathrm{a}}$ & Ref. $5^{\mathrm{e}}$ & This work ${ }^{\mathrm{a}}$ & & \\
\hline \multicolumn{9}{|c|}{ Simplified CGC } \\
\hline 1 & Ti-Me & Ethylene & -25.2 & $-24.1(-22.7)$ & 4.0 & 4.1(7.4) & $-167.9($ n.d) & $2.275(2.222)$ \\
\hline 2 & Ti-Me & 2,1-styrene & -31.9 & $-31.0(-30.3)$ & 7.8 & 11.7(16.7) & $-279.7(-349.5)$ & $2.270(2.123)$ \\
\hline 3 & Ti-E & Ethylene & n.d & $-16.1(-13.6)$ & n.d & n.a.(5.5) & n.d. $(-188.9)$ & n.d. $(2.290)$ \\
\hline 4 & Ti-E & 2,1-styrene & n.d & $-20.8(-20.9)$ & n.d & $9.6(14.8)$ & $-209.5(-299.8)$ & $2.350(2.153)$ \\
\hline 5 & Ti-St & Ethylene & -19.0 & $-7.1(-4.4)$ & 1.9 & $6.7(9.8)$ & $-95.9(\mathrm{n} . \mathrm{d})$ & $2.441(2.333)$ \\
\hline 6 & $\mathrm{Ti}-\mathrm{St}$ & 2,1-styrene & -20.9 & $-10.4(-8.8)$ & 4.1 & $9.8(13.9)$ & $-214.8(-255.7)$ & $2.163(2.151)$ \\
\hline \multicolumn{9}{|c|}{ Real CGC } \\
\hline 7 & Ti-Me & Ethylene & n.d & $-16.4(-17.0)$ & n.d & $2.9(7.0)$ & $-205.5(-299.9)$ & $2.247(2.180)$ \\
\hline 8 & Ti-Me & 2,1-styrene & n.d & $-19.7(-20.6)$ & n.d & $10.6(16.0)$ & $-284.1(-352.2)$ & $2.135(2.106)$ \\
\hline 9 & Ti-E & Ethylene & n.d & $-9.3(-9.4)$ & n.d & n.a.(6.0) & n.d. $(-179.8)$ & n.d. (2.325) \\
\hline 10 & Ti-E & 2,1-styrene & n.d & $-11.9(-12.5)$ & n.d & $8.9(14.6)$ & $-214.4(-280.9)$ & $2.210(2.181)$ \\
\hline 11 & Ti-St & Ethylene & n.d & $-0.7(+1.8)$ & n.d & $1.1(9.6)$ & $-170.4(-219.2)$ & $2.269(2.252)$ \\
\hline 12 & $\mathrm{Ti}-\mathrm{St}$ & 2,1-styrene & n.d & $-0.3(-0.7)$ & n.d & $9.3(16.1)$ & $-225.6(-257.8)$ & $2.185(2.202)$ \\
\hline
\end{tabular}

BP86/LANL2DZ (B3LYP/LANL2DZ).

${ }^{\mathrm{b}}$ Definition is shown in Fig. 1.

${ }^{\mathrm{c}}$ Complexation energy $\left(\Delta E_{\text {comp }}\right)$ is defined as the difference between the electronic energy of the $\pi$-complex and the summation of electronic energies of separated reactants.

${ }^{\mathrm{d}}$ Electronic energy barrier $\left(\Delta E_{\text {comp }}\right)$ is defined as the difference between the electronic energy of the transition state and the electronic energy of the corresponding $\pi$-complex.

${ }^{\mathrm{e}}$ BP86/DNP level. See Ref. 5 for details.

${ }_{\mathrm{f}}^{\mathrm{f}}$ Transition structure distance between the carbon atom of the alkyl growing polymer chain attached to the titanium atom and the closest ethylene carbon atom to $\mathrm{C} \alpha$. See Ref. 1 for details. 
TABLE II. Effect of the polarization functions on the ethylene and secondary styrene monomer insertion into the Ti-Me and Ti-St species for the CGC-simplified catalyst. Calculations were done at BP86 level using a basis set without (LANL2DZ) and with (DZVP) polarization functions. These energies can be compared with those obtained by Yang et al. shown in Table I. Energies are given in $\mathrm{kcal} / \mathrm{mol}$.

\begin{tabular}{cccccc}
\hline \hline & & \multicolumn{2}{c}{$\Delta E_{\text {comp }}{ }^{c}$} & \multicolumn{2}{c}{$\Delta E^{\mathrm{d}}$} \\
\cline { 3 - 6 } Species $^{\mathrm{a}}$ & \multirow{2}{*}{ Monomer $^{\mathrm{b}}$} & LANL2DZ & DZVP $^{\mathrm{a}}$ & LANL2DZ & DZVP \\
\hline Ti-Me & Ethylene & -24.1 & -25.0 & 4.1 & 3.5 \\
Ti-Me & $2,1-$-styrene & -31.0 & -31.0 & 11.7 & 9.4 \\
Ti-St & Ethylene & -7.1 & -6.1 & 6.7 & 5.4 \\
Ti-St & 2,1 -styrene & -10.4 & -8.7 & 9.8 & 8.2 \\
\hline \hline
\end{tabular}

${ }^{\mathrm{a}}$ Basis set with polarization functions for all atoms except for hydrogen. See Ref. 10 for more details.

${ }^{\mathrm{b}}$ Definition is shown in Fig. 1.

${ }^{\mathrm{c}}$ Complexation energy $\left(\Delta E_{\text {comp }}\right)$ is defined as the difference between the electronic energy of the $\pi$-complex and the summation of electronic energies of separated reactants.

${ }^{d}$ Electronic energy barrier $\left(\Delta E_{\text {comp }}\right)$ is defined as the difference between the electronic energy of the transition state and the electronic energy of the corresponding $\pi$-complex.

very similar for both models. Thus, it seems that the use of a simplified CGC model is a reliable approach to monomer insertion into a constrained geometry catalyst.

Experimentally, a sharp decrease in catalytic activity is observed as the styrene concentration in the reactor feed is increased. ${ }^{14}$ This result suggests the formation of "dormant species" when styrene is present in the reactor. ${ }^{14}$ In our theoretical study dormant species are attributed to Ti-St species (Fig. 1), which have an ethylene insertion energy barrier $(6.7 \mathrm{kcal} / \mathrm{mol}$, entry 5 in Table I) higher than two consecutive ethylene insertions (entry 3 in Table I). However, the energy barrier calculated by Yang et al. ${ }^{5}$ is unable to explain this experimental finding. Thus, their calculated energy barrier for an ethylene insertion after a styrene insertion is $1.9 \mathrm{kcal} / \mathrm{mol}$, which is lower than two consecutive ethylene insertions $(4.0 \mathrm{kcal} / \mathrm{mol})$. Therefore, the activity of the catalyst should increase rather than decrease in the presence of styrene, which is in clear opposition to the experimental findings. ${ }^{14,15}$

The formation of styrene blocks is not experimentally confirmed according to the ${ }^{13} \mathrm{C}$-NMR spectrum of the copolymers. ${ }^{14}$ Our barriers are qualitatively consistent with the complete lack of formation of styrene blocks (9.6 for St insertion into Ti-E and $9.8 \mathrm{kcal} / \mathrm{mol}$ for St insertion into Ti-St, entries 4 and 6, respectively, in Table I). This difference is even greater when energy barriers for the real CGC are taken into account $(8.9$ and $9.3 \mathrm{kcal} / \mathrm{mol}$, entries 10 and 12, respectively, in Table I).However, the energy barriers obtained by other authors ${ }^{5}$ for a styrene insertion after an ethylene or a styrene insertion are 7.8 and $4.1 \mathrm{kcal} / \mathrm{mol}$, respectively. Therefore, according to these authors the formation of styrene blocks should be a likely process, since St-St insertions are apparently easier than E-St ones.

An activation energy for the E-St copolymerization process using the CGC catalyst of about $13.1-13.4 \mathrm{kcal} / \mathrm{mol}$ has been experimentally determined. ${ }^{14,15}$ Averaging electronic energy barriers for the insertion processes, a value in the range of $8.1-12.2 \mathrm{kcal} / \mathrm{mol}$ is obtained (depending on the CGC model and theoretical method selected). The energy barriers for the E-St copolymerization using the "cationic model" correlate reasonably well with the activation energies experimentally found, in spite that cocatalyst and solvent effects have been neglected. In addition, these values are closer to the experimental activation energy than those reported by other authors. ${ }^{5}$

Interactions between a phenyl group belonging to the growing polymer chain and the titanium metal in $\pi$-complexes and transition structures have been obtained by other authors. ${ }^{5}$ This was referred to as a "phenyl-agostic" interaction and described in terms of the distance between $\mathrm{Ti}$ and the centroid of the phenyl group, which is in the range of 3.11-3.20 ̊. However, we have obtained more stable structures than those showing phenyl-agostic interactions. Our structures have a larger Ti-phenyl centroid distance, in the range 3.70-4.30 ̊. We have only found these phenylagostic structures to be the most stable in the case of the styrene insertion product, but not for $\pi$-complexes and transition structures, since when the monomer is coordinated to the metal center, the phenyl-agostic interaction is lost. We have also attempted to fully optimize $\pi$-complexes by forcing a phenyl-agostic interaction using the BP86/LANL2DZ and BP86/DZVP methods, converging all geometries to structures without this type of interaction.

To clarify the differences described above, we have examined the different methods for locating transition state (TS) structures used by us and other authors. ${ }^{5}$ As it can be noted in that work $^{5}$ the reaction coordinates for all the transition structures are the same $\left(\mathrm{C}_{2}-\mathrm{C}_{\alpha}=2.500 \AA\right)$. In our calculations, the $\mathrm{C}_{2}-\mathrm{C}_{\alpha}$ distance was shorter especially for the styrene insertion, as can be seen in Table I $\left(\mathrm{C}_{2}-\mathrm{C}_{\alpha}\right.$ $=2.220-2.440 \AA$ for the ethylene insertion and $\mathrm{C}_{2}-\mathrm{C}_{\alpha}$ $=2.120-2.160 \AA$ for the styrene insertion). These $\mathrm{C}_{2}-\mathrm{C}_{\alpha}$ distances are consistent with those reported by other authors $\left(\mathrm{C}_{2}-\mathrm{C}_{\alpha}=2.130 \AA\right) .{ }^{16}$ Further, by comparing geometrical parameters such as $\mathrm{C}_{2}-\mathrm{C}_{\alpha}, \mathrm{C}_{1}-\mathrm{C}_{2} \mathrm{C}_{1}-\mathrm{Ti}$, and $\mathrm{C}_{\alpha}-\mathrm{Ti}$ distances, it could be inferred that the transition structures obtained by Yang et $a l .{ }^{5}$ are not truly saddle points in the potential energy surface, and therefore their associated energy barriers are very low. This may be explained by the fact that they performed "coordinate driving" calculations to find a TS without verifying the nature of the "saddle points" obtained through the number of negative eigenvalues. It is well known that this method of locating TSs fails when the TSs have reaction vectors not dominated by only one internal variable. ${ }^{11,17}$ For processes of olefin insertion into this type of CGC species, at least four internal variables participate in the TS $\left(\mathrm{C}_{2}-\mathrm{C}_{\alpha}, \mathrm{C}_{1}-\mathrm{C}_{2} \mathrm{C}_{1}-\mathrm{Ti}\right.$, and $\left.\mathrm{C}_{\alpha}-\mathrm{Ti}\right)$. In our case, transition state geometries were obtained by the STQN method to locate an estimated saddle point in the path from reactant to product. Subsequently, this guess saddle point was completely optimized following the negative eigenvector. Frequency calculations were then performed to check the nature of the stationary points found. Thus, transition states were characterized by exactly one imaginary frequency (see Table I) and the corresponding eigenvector visualized. ${ }^{11}$ In our opinion taking into account the present state of art in 
computer modeling, the coordinate driving methodology should only be used to locate guess structures which must be subsequently optimized following the negative eigenvector. ${ }^{17}$

\section{CONCLUSION}

A DFT study of the ethylene-styrene copolymerization mechanism with a simplified and real CGC model catalysts has been carried out. We have observed that the energy barriers of the monomer insertion process are slightly affected by the presence of the bulky ligands in the real CGC model compared to the simplified CGC model. However, the complexation step are influenced by the presence of the bulky ligands due to steric interactions between the incoming monomer and the $t$-butyl ligand of the catalyst. On the other hand, we have found that the polarization functions have a slight effect (less than $2 \mathrm{kcal} / \mathrm{mol}$ ) on both complexation energies and energy barriers for the monomer insertion in agreement with other authors. ${ }^{12,13}$

The calculated energy profiles for the ethylene and styrene insertions are in agreement with the experimental findings such as no formation of St-St blocks and the decrease in the catalyst activity when the styrene concentration increases in the monomer feed.

In addition, the use of an interpolation method such as QST3 (Ref. 11) is highly recommended to obtain a suitable estimate TS followed by complete optimization along the negative eigenvector and a frequency analysis. Thus, it seems to be that the energy barriers reported by Yang et al. ${ }^{5}$ are too low. This is possibly due to the coordinate driving method used to obtain them.

\section{ACKNOWLEDGMENTS}

Thanks are given to CICYT (Grant MAT2002-01242) for funding this investigation. Sonia Martinez was awarded a fellowship by the MECD.

${ }^{1}$ A. Muñoz-Escalona, V. Cruz, N. Mena, S. Martínez, and J. MartínezSalazar, Polymer 43, 7017 (2002).

${ }^{2}$ S. Martínez, V. Cruz, A. Muñoz-Escalona, and J. Martínez-Salazar, Polymer 44, 295 (2002).

${ }^{3}$ M. T. Expósito, S. Martínez, J. Ramos, V. Cruz, M. López, A. MuñozEscalona, N. Haider, and J. Martínez-Salazar, Polymer 45, 9029 (2004).

${ }^{4}$ S. Martínez, M. T. Expósito, J. Ramos, V. Cruz, M. C. Martínez, M. López, A. Muñoz-Escalona, and J. Martínez-Salazar, J. Polym. Sci. Part A: Chem. (to be published).

${ }^{5}$ S. H. Yang, W. H. Jo, and S. K. Noh, J. Chem. Phys. 119, 1824 (2003).

${ }^{6}$ J. Ramos, V. Cruz, A. Muñoz-Escalona, and J. Martinez-Salazar, Polymer 41, 6161 (2000).

${ }^{7}$ J. Ramos, A. Muñoz-Escalona, V. Cruz, and J. Martinez-Salazar, Polymer 44, 2177 (2003).

${ }^{8}$ M. J. Frisch, G. W. Trucks, H. B. Schlegel et al., GAussian98 (Revision A.1) (Gaussian Inc. Pittsburgh, PA, 1998).

${ }^{9}$ P. J. Hay and W. R. Wadt, J. Chem. Phys. 82, 270 (1985).

${ }^{10}$ N. Godbout, D. R. Salahub, J. Andzelm, and E. Wimmer, Can. J. Chem. 70, 560 (1992).

${ }^{11} \mathrm{~F}$. Jensen, Introduction to Computational Chemistry (Wiley, New York, 1999), pp. 327-338.

${ }^{12}$ D. G. Musaev, R. D. J. Froese, and K. Morokuma, Organometallics 17, 1850 (1998).

${ }^{13}$ G. Talarico, A. N. J. Blok, T. K. Woo, and L. Cavallo, Organometallics 21, 4939 (2002).

${ }^{14}$ F. G. Sernetz, R. Mülhaupt, and R. M. Waymouth, Macromol. Chem. Phys. 197, 1071 (1996).

${ }^{15}$ T. A. Sukhova, A. N. Panin, O. N. Babkina, and N. M. Bravaya, J. Polym. Sci., Part A: Polym. Chem. 37, 1083 (1999).

${ }^{16}$ G. Lanza, I. L. Fragalà, and T. J. Marks, Organometallics 21, 5594 (2002).

${ }^{17}$ H. B. Schlegel, J. Comput. Chem. 24, 1514 (2003). 